\title{
25.
}

\section{Théorèmes sur les courbes de troisième degré.}

\author{
(Par Mr. George Salmon à Dublin.)
}

Le théorème suivant me paraît très fécond en conséquences pour les courbes de troisieme ordre.

1. Si d'un point quelconque $(O)$ d'une telle courbe on tire à la courbe quatre tangentes $(O A, O B, O C, O D)$, la fonction enharmonique de ce faisceau $\left(\frac{\sin A O B \sin C O D}{\sin A O C \sin B O D}\right)$ est constante. Par conséquent chaque courbe de troisième ordre a une caractéristique numérique qui ne change pas par la projection en quelqu'autre transformation linéaire.

Car celte fonction ne change pas si l'on passe au point consécutif $(\boldsymbol{P})$ de la courbe. Qu'on mène quatre tangentes du point $\boldsymbol{P}$ : chaque tangente rencontre la tangente consécutive à son point de contact avec la courbe: mais il est connu que les six points $\boldsymbol{O}, \boldsymbol{P}, \boldsymbol{A}, \boldsymbol{B}, \boldsymbol{C}, \boldsymbol{D}$ sont sur la même conique.

On peut aussi démontrer algébriquement la même proposition, en formant l'expression générale de la fonction enharmonique; et on trouvera que cette fonction dépend seulement du rapport $S^{3}: T^{2} ;$ où $S$ et $T$ sont les fonctions qui (comme M. Aronhold l'a fait voir) ne changent pas par des transformations linéaires.

Par cette méthode on arrive aussi facilement au théorème de M. Aronhold, savoir que la condition $(R)$, pour qu'une courbe de troisième ordre puisse avoir un point double, est de la forme

$$
\boldsymbol{R}=\boldsymbol{S}^{3}-\boldsymbol{T}^{2} \text {. }
$$

Car l'équation biquadratique qui donne les quatre tangentes d'un point quelconque de la courbe, et qui est de la forme

$$
\boldsymbol{A} x^{4}+4 B x^{3} y+6 C x^{2} y^{2}+4 D x y^{3}+E y^{4}=0,
$$

peut être formée sans difficulté. Or la condition pour les deux racines de cette équation d'être égales, est

$$
\left(A E-4 B D+3 C^{2}\right)^{3}=27\left(A C E+2 B C D-A D^{2}-E B^{2}-C^{3}\right)^{2}
$$

ce qui donne directement la formule de M. Aronhold.

Trois racines de l'équation seront égales et la courbe aura un point de rebroussement, si $\boldsymbol{S}=\mathbf{0}, \boldsymbol{T}=\mathbf{0}$. 
Par notre méthode on peut aussi former aisément les fonctions $\boldsymbol{S}, \boldsymbol{T}$, que M. Aronhold n'a pas données explicitement.

Soit l'équation de la courbe $a_{1} x^{3}+3 a_{2} x^{2} y+3 b_{1} x y^{2}+b_{2} y^{3}+3 a_{3} x^{2} z+b f x y z+3 b_{3} y^{2} z+3 c_{1} x z^{2}+3 c_{2} y z^{2}$ $+c_{3} z^{3}=0$

on obtient

$$
S=f^{4}-2 f^{2}\left(b_{1} c_{1}+c_{2} a_{2}+a_{3} b_{3}\right)+3 f\left(a_{2} c_{1} b_{3}+a_{3} b_{1} c_{2}\right)-f a_{1} b_{2} c_{3}
$$

$+f\left(a_{1} b_{3} c_{2}+b_{1} c_{3} a_{2}+c_{1} a_{3} b_{2}\right)-\left(a_{1} c_{1} b_{3}^{2}+b_{2} c_{2} a_{3}^{2}+a_{1} b_{1} c_{2}^{2}+c_{3} b_{3} a_{2}^{2}+a_{3} c_{3} b_{1}^{2}+a_{2} b_{2} c_{1}^{2}\right)$ $+\left(a_{1} c_{1} b_{2} c_{2}+a_{1} b_{1} c_{3} b_{3}+a_{2} b_{2} a_{3} c_{3}\right)-\left(a_{2} c_{2} b_{1} c_{1}+b_{1} c_{1} a_{3} b_{3}+a_{2} c_{2} a_{3} b_{3}\right)+a_{2}^{2} c_{2}^{2}+b_{3}^{2} a_{3}^{2}+c_{1}^{2} b_{1}^{2}$, $\boldsymbol{T}=-8 f^{6}+24 f^{4}\left(b_{1} c_{1}+a_{2} c_{2}+a_{3} b_{3}\right)-36 f^{3}\left(a_{2} b_{3} c_{1}+a_{3} b_{1} c_{2}\right)-12 f^{3}\left(a_{1} b_{3} c_{2}+a_{2} b_{1} c_{3}\right.$ $\left.+a_{3} b_{2} c_{1}\right)-20 a_{1} b_{2} c_{3} f^{3}-24 f^{2}\left(b_{1}^{2} c_{1}^{2}+a_{2}^{2} c_{2}^{2}+a_{3}^{2} b_{3}^{2}\right)+12 f^{2}\left(a_{1} c_{1} b_{3}^{2}+a_{3} c_{3} b_{1}^{2}+b_{3} c_{3} a_{2}^{2}\right.$ $\left.+b_{2} c_{2} a_{3}^{2}+a_{2} b_{2} c_{1}^{2}+a_{1} b_{1} c_{2}^{2}\right)+36 f^{2}\left(a_{1} b_{2} c_{1} c_{2}+b_{2} c_{3} a_{2} a_{3}+a_{1} c_{3} b_{1} b_{3}\right)-12 f^{2}\left(a_{2} b_{1} c_{1} c_{2}\right.$ $\left.+b_{3} c_{2} a_{2} c_{3}+a_{3} c_{1} b_{1} b_{3}\right)+36 f\left(a_{3} b_{1} c_{2}+a_{2} b_{3} c_{1}\right)\left(a_{2} c_{2}+a_{3} b_{3}+b_{1} c_{1}\right)+12 f\left(c_{3} c_{2} b_{1} a_{2}^{2}\right.$ $\left.+c_{1} c_{3} a_{2} b_{1}^{2}+a_{1} a_{2} b_{3} c_{2}^{2}+b_{1} b_{2} c_{1} a_{3}^{2}+a_{1} a_{2} c_{2} b_{3}^{2}+b_{1} b_{2} a_{3} c_{1}^{2}\right)-60 f\left(a_{2} a_{3} b_{1} b_{3} c_{3}+a_{1} b_{1} b_{3} c_{1} c_{2}\right.$ $\left.+a_{2} a_{3} b_{2} c_{1} c_{2}\right)+12 f a_{1} b_{2} c_{3}\left(a_{3} b_{3}+b_{1} c_{1}+a_{2} c_{2}\right)-24 f\left(a_{1} a_{2} b_{3}^{2} c_{3}+a_{3}^{2} b_{1} b_{2} c_{3}+b_{1}^{2} a_{1} c_{2} c_{3}\right.$ $\left.+c_{1}^{2} a_{1} b_{2} b_{3}+c_{2}^{2} a_{1} a_{3} b_{2}+a_{2}^{2} b_{2} c_{1} c_{3}\right)+6 a_{1} b_{2} c_{3}\left(b_{1} c_{2} a_{3}+c_{1} a_{2} b_{3}\right)+6\left(a_{2} a_{3} b_{1}^{2} c_{2} c_{3}\right.$ $\left.+a_{1} a_{2} b_{3}^{2} c_{1} c_{2}+a_{2}^{2} b_{1} b_{3} c_{1} c_{3}+a_{3}^{2} b_{1} b_{2} c_{1} c_{2}+a_{2} a_{3} b_{2} b_{3} c_{1}^{2}+a_{1} a_{3} b_{1} b_{3} c_{2}^{2}\right)+a_{1}^{2} b_{2}^{2} c_{3}^{2}$ $+24\left(a_{1} c_{1} b_{1}^{2} c_{2}^{2}+a_{1} b_{1} b_{3}^{2} c_{1}^{2}+b_{2} c_{2} a_{2}^{2} c_{1}^{2}+a_{2} b_{2} a_{3}^{2} c_{2}^{2}+a_{3} c_{3} a_{2}^{2} b_{3}^{2}+b_{3} c_{3} a_{3}^{2} b_{1}^{2}\right)$ $+18\left(a_{2} a_{3} b_{1} b_{2} c_{1} c_{3}+a_{1} a_{2} b_{1} b_{3} c_{2} c_{3}+a_{1} a_{3} b_{2} b_{3} c_{1} c_{2}\right)-12\left(a_{1} a_{2} b_{1} c_{2}^{3}+a_{2} b_{1} b_{2} c_{1}^{3}+a_{1} a_{3} c_{1} b_{3}^{3}\right.$ $\left.+b_{2} b_{3} c_{2} a_{3}^{3}+b_{3} c_{2} c_{3} a_{2}^{3}+a_{3} c_{1} c_{3} b_{1}^{3}\right)+4\left(a_{1} b_{1}^{3} c_{3}^{2}+a_{1}^{2} b_{2} c_{2}^{3}+a_{2}^{3} b_{2} c_{3}^{2}+a_{3}^{3} b_{2}^{2} c_{3}+a_{1} b_{2}^{2} c_{1}^{3}\right.$ $\left.+a_{1}^{2} b_{3}^{3} c_{3}\right)-3\left(a_{2}^{2} b_{1}^{2} c_{3}^{2}+a_{1}^{2} b_{3}^{2} c_{2}^{2}+a_{3}^{2} b_{2}^{2} c_{1}^{2}\right)-6\left(a_{1}^{2} b_{2} b_{3} c_{2} c_{3}+a_{1} a_{3} b_{2}^{2} c_{1} c_{3}+a_{1} a_{2} b_{1} b_{2} c_{3}^{2}\right)$ $+8\left(b_{1}^{3} c_{1}^{3}+a_{2}^{3} c_{2}^{3}+a_{3}^{3} b_{3}^{3}\right)-27\left(a_{3}^{2} b_{1}^{2} c_{1}^{2}+a_{2}^{2} b_{3}^{2} c_{1}^{2}\right)-12\left(a_{1} a_{2} b_{2} c_{1} c_{2}^{2}+a_{1} b_{2} b_{1} c_{1}^{2} c_{2}\right.$ $\left.+a_{2}^{2} a_{3} b_{2} c_{2} c_{3}+a_{2} a_{3}^{2} b_{2} b_{3} c_{3}+a_{1} a_{3} b_{1} b_{3}^{2} c_{3}+a_{1} b_{1}^{2} b_{3} c_{1} c_{3}\right)-6 a_{1} a_{2} b_{1} c_{1} a_{3} b_{3}-12\left(a_{2} b_{1}^{2} c_{1}^{2} c_{2}\right.$ $\left.+a_{3} b_{1}^{2} c_{1}^{2} b_{3}+a_{2}^{2} b_{1} c_{1} c_{2}^{2}+a_{2}^{2} a_{3} b_{3} c_{2}^{2}+a_{3}^{2} b_{1} c_{1} b_{3}^{2}+a_{2} a_{3}^{2} b_{3}^{2} c_{2}\right)$

et

$$
R=T^{2}-64 S^{3}
$$

Reprenons maintenant le théorème (1.). On en tire la proposition suivante.

2. Si à deux points de la courbe $\boldsymbol{P}, \boldsymbol{Q}$, on mène des tangentes $p_{1}, p_{2}, p_{3}, p_{4}$, $q_{1}, q_{2}, q_{3}, q_{4}$, les points d'intersection, $p_{1} q_{1}, p_{2} q_{2}, p_{3} q_{3}, p_{4} q_{4}$ se trouveront sur une conique qui passe aussi par les points $\boldsymbol{P}, \boldsymbol{Q}$. Il existe quatre coniques de cette sorte, dont chacune passe par les points $\boldsymbol{P}, \boldsymbol{Q}$ et par quatre des seize points d'intersection des tangentes.

3. Soient $\boldsymbol{P}, \boldsymbol{Q}$ les deux points imaginaires à l'infini sur un cercle quelconque; alors, suivant la définition de M. Plücker (3.), une courbo circulaire de troisième degré a seize foyers, disposés sur quatre cercles. 
Seulement quatre de ces foyers sont réels. La courbe a en outre un föer double.

Les théorèmes (2. et 3.) sont dù à M. Hart qui les a obtenus comme suit. D'abord la propriété caractéristique des ovales de Descarles est $a \varrho_{1}+b \varrho_{2}=c$, où $\varrho_{1}, \varrho_{2}$ sont les distances de quelque point de la courbe à deux points fixes. M. Chasles a démontré que la courbe a de plus un foyer tel que $a_{1} \varrho_{1}+b_{1} \varrho_{3}=c_{1}$, et M. Hart a trouvé l'extension suivante de ce théorème.

,Soit $a \varrho_{1} \pm b \varrho_{2} \pm c \varrho_{3}=0$ la propriété caractéristique d'une courbe; alors cette courbe sera du quatrième ordre, et aura de plus un foyer tel que $a_{1} \varrho_{1} \pm b_{1} \varrho_{2} \pm c_{1} \varrho_{4}=0$. Les quatre foyers seront disposés sur un cercle; et si $a \pm b \pm c=0$, la courbe ne sera que du troisième degrè." Une courbe de troisième degré aura quatre foyers, pourvu que les termes les plus élévés en $x$ et $y$ soient divisibles par $x^{2}+y^{2}$.

Le problème suivant "Les quatre foyers étant donnés: décrire la courbe" admet deux solutions. Soient les quatre foyers $A, B, C, D$. Si $A B, C D$, s'entrecoupent en $\boldsymbol{O}$; l'équation de la courbe sera, ou

$$
\begin{aligned}
& (\boldsymbol{B O}+\boldsymbol{O C}) \varrho_{1}+(\boldsymbol{A O}-\boldsymbol{O C}) \varrho_{2}=\boldsymbol{A B} \varrho_{3} \\
& (\boldsymbol{B O}-\boldsymbol{O D}) \varrho_{1}+(\boldsymbol{A O}+\boldsymbol{O D}) \varrho_{2}=\boldsymbol{A B} \varrho_{4},
\end{aligned}
$$

ou

$$
\begin{aligned}
& (B O-O C) \varrho_{1}+(A O+O C) \varrho_{2}=A B \varrho_{3} \\
& (B O+O D) \varrho_{1}+(A O-O D) \varrho_{2}=A B \varrho_{4} .
\end{aligned}
$$

De ces équations on tire $\boldsymbol{C D}\left(\varrho_{1} \mp \varrho_{2}\right)=\boldsymbol{A B}\left(\varrho_{3} \mp \varrho_{t}\right)$, c'est à dire, la courbe est le lieu géométrique de l'intersection de deux coniques dont les foyers sont donnès.

Les deux courbes s'entrecoupent à angles droits au centre du cercle et aux trois points $\boldsymbol{O}, \boldsymbol{P}, \boldsymbol{Q}$, qui sont les intersections des droites $\boldsymbol{A B}, \boldsymbol{C D}$; $\boldsymbol{A C}, \boldsymbol{B D} ; \boldsymbol{A D}, \boldsymbol{B C}$. Les tangentes à ces points sont parellèles aux bisecteurs de l'angle $\boldsymbol{A O C}$. On voit aisément que l'asymptote de la courbe est aussi parallèle à l'une ou l'autre de ces bisecteurs. Donc, au moyen de la projection on obtient cette proposition:

Soient $\boldsymbol{P}, \boldsymbol{Q}, \boldsymbol{R}$ les trois points dans lesquels une droite rencontre une courbe de troisième ordre, et menez les tangentes $p_{1}, p_{2}, p_{3}, p_{4}, q_{1}, q_{2}, q_{3}, q_{3}$ : alors les intersections des droites qui joignent $\left(p_{1} q_{1}-p_{2} q_{2}\right),\left(p_{3} q_{3}-p_{4} q_{4}\right)$; $\left(p_{1} q_{1}, p_{3} q_{3}\right)\left(p_{2} q_{2}, p_{4} q_{4}\right) ;\left(p_{1} q_{1}, p_{4} q_{4}\right)\left(p_{2} q_{2}, p_{3} q_{3}\right)$ seront des points de contact des tangentes qui passent par $\boldsymbol{R}$ : le quatrième point de contact sera le pôle de $\boldsymbol{P Q}$ pour la conique qui passe par $\boldsymbol{P}, \boldsymbol{Q}, p_{1} q_{1}, p_{2} q_{2}, p_{3} q_{3}, p_{4} q_{4}$.

Trinity College Dublin July 24. 1851. 